\title{
Establishing a standardized FUO emergency department: design and practice in dealing with COVID-19
}

\author{
Tongtong Chen ${ }^{1 \#}$, Xudong Ma ${ }^{2 \#}$, Shuai Zhou ${ }^{3}$, Hanqi Wang ${ }^{1}$, Yaling Pan ${ }^{1}$, Liuping Chen ${ }^{1}$, Haiying Lv ${ }^{1}$, \\ Yong $\mathrm{Lu}^{1}$
}

${ }^{1}$ Department of Radiology, Ruijin Hospital/Lu Wan Branch, School of Medicine, Shanghai Jiaotong University, Shanghai, China; ${ }^{2}$ National Health and Family Planning Commission of the People's Republic of China, Beijing, China; ${ }^{3}$ Division of Medical Affairs, Ruijin Hospital, School of Medicine, Shanghai Jiaotong University, Shanghai, China

Contributions: (I) Conception and design: Y Lu; (II) Administrative support: X Ma; (III) Provision of study materials or patients: Y Lu, T Chen; (IV) Collection and assembly of data: S Zhou, T Chen, H Wang; (V) Data analysis and interpretation: Y Pan, L Chen, H Lv; (VI) Manuscript writing: All authors; (VII) Final approval of manuscript: All authors.

\#These authors contributed equally to this paper.

Correspondence to: Yong Lu. Department of Radiology, Ruijin Hospital/Lu Wan Branch, School of Medicine, Shanghai Jiaotong University, No. 149, South Chongqing Road, Shanghai, China. Email: 18917762053@163.com.

Background: Since the outbreak of COVID-19 in January, 2020, the fever of unknown origin (FUO) emergency department has become the first station for disease prevention and identification in hospitals. Establishing a standardized FUO emergency department within a short period of time has become the key to preventing and controlling COVID-19 in densely populated Chinese cities.

Methods: Based on the lean management model, the research group established a process of less-link visits, which sees reduced contact between patients and physicians during diagnosis and treatment, and zerocontact consultation through lean workflow and value stream analysis. Three steps were implemented to improve the operations of the FUO emergency department: the rapid establishment of an isolation zone, the refinement of duty and protection configuration, and the use of Internet and technology to establish a fullprocess follow-up consultation system.

Results: (I) Tests related to COVID-19 screening are all completed in the FUO emergency department; (II) 12 new isolated observation rooms have been built; (III) hospital visiting time, waiting time for consultation, and the time from pre-examination to virus screening has been shortened from 18 to 8 hours, from 2 hours to 10 minutes, and from 34 to 3 hours, respectively; (IV) the transfer distance has been shortened from 450 to $20 \mathrm{~m}$, and the observation time has been shortened from 72 to 26 hours. The median waiting time for image examination has been reduced from 40 to 3 minutes, and the moving distance has been shortened from 800 to $10 \mathrm{~m}$; (V) the diagnosis and treatment process is facilitated by $5 \mathrm{G}$, achieving zero contact between doctors and patients.

Conclusions: Through the implantation of information technology, the local transformation of the site, the rational allocation of medical teams and the planned distribution of protective equipment, in a short period of time, individual medical institutions can set up a safe FUO emergency department to provide 24hour screening and detention services. Establishing an FUO emergency department with lean management and realizing the management approach of combining daily operation with prevention and control could help China and other countries to handle the outbreak of fulminant infectious diseases.

Keywords: COVID-19; FUO emergency department; Infectious disease; Lean management model; internet + technology

Submitted Apr 03, 2020. Accepted for publication Jun 18, 2020.

doi: 10.21037/atm-20-4329

View this article at: http://dx.doi.org/10.21037/atm-20-4329 


\section{Introduction}

Since the outbreak of coronavirus disease 2019 (COVID-19) in January, 2020, the fever of unknown origin (FUO) emergency department has become the first line in preventing and identifying the disease in hospitals. In densely populated cities in China, establishing a standardized FUO emergency department within a short period of time (one week) has become central in the approach to preventing and controlling COVID-19.

Following the severe acute respiratory syndrome (SARS) epidemic in 2003, the Ministry of Health of China instructed tertiary hospitals and some regional hospitals to set up 24-hour FUO emergency departments (1). In the current COVID-19 epidemic, nosocomial transmission has proved to be a key way in which disease is transmitted. One of the typical symptoms of COVID-19 is fever. The establishment of the FUO department is beneficial to quickly concentrate patients with fever symptoms and isolate them from general emergency patients, which is an effective way to prevent nosocomial infection.

During the time of the COVID-19 outbreak, the FUO emergency department has become hospitals' first line of defense and the most important department for receiving and screening suspected cases (2). With limited space and time at their disposal, hospitals have been tasked with improving inspection facilities in the FUO emergency department, implementing effective regional isolation, and avoiding nosocomial infection as the key approaches to preventing and controlling COVID-19. Focusing on Ruijin Hospital affiliated to the School of Medicine of Shanghai Jiaotong University, a large comprehensive medical institution in the center of Shanghai, as an example, this study investigates how effective prevention and control management of the FUO emergency department can be implemented in the shortest possible amount of time.

Ruijin Hospital is a university-affiliated general hospital located in central Shanghai. Founded more than 110 years ago, the hospital has more than 2,300 open beds and sees more than 12,000 outpatients a day. The bustling area around the hospital, which contains more than 40 buildings, has changed very little in the past 70 years. The four main ward buildings are connected by corridors, while the other buildings are stand alone.

In accordance with the requirements introduced by the former Ministry of Health in 2003, Ruijin Hospital set up a 24-hour FUO emergency department on the first floor of the old outpatient building. At that time, the structure of the 40-year-old building, with 3 isolated, narrow observation rooms, a clinical laboratory big enough to accommodate only routine examinations, and access to only mobile X-ray equipment, presented the main challenges to the FUO emergency department (Figure 1). At the end of 2019, reconstruction work on the emergency building began; the FUO emergency department will be converted into a transitional pediatric emergency department.

In 2006, the first patients arrived at the hospital's Infection and Respiratory Disease Building, which, with 400 beds and an independent virology laboratory, was the largest medical building for infectious diseases in downtown Shanghai at that time. Back then, the building was equipped with only two negative pressure wards, and there were no working areas with independent central air conditioning for the treatment of respiratory infections.

In the event of a new COVID-19 outbreak occurring, Ruijin Hospital must upgrade the capacity of its FUO emergency department as quickly as possible to provide timely diagnosis, treatment, and medical observation for the continuous influx of patients with fever, and to reduce the threat of nosocomial infection for medical staff (3).

\section{Methods}

Based on the lean management model, the research group established a process of less-link visits, which sees reduced contact between patients and physicians during diagnosis and treatment, and zero-contact consultation through lean workflow and value stream analysis. Three steps were implemented to improve the operation of the FUO emergency department. First, the space was rapidly integrated to establish an isolation zone. Second, duties and protection configuration were refined. Third, a full-process follow-up consultation system was established using the Internet and technology.

\section{Re-planning and rapid implementation of the FUO emergency department}

\section{Rapidly integrating space to establish a standard febrile} emergency department

Between January and November 2019, on average, the FUO emergency department received 20 patients with fever per day, most of whom were influenza patients. Vacating space and establishing an FUO emergency department that met the standard in a limited amount of time presented the most significant problems for the hospital (4). In particular, 


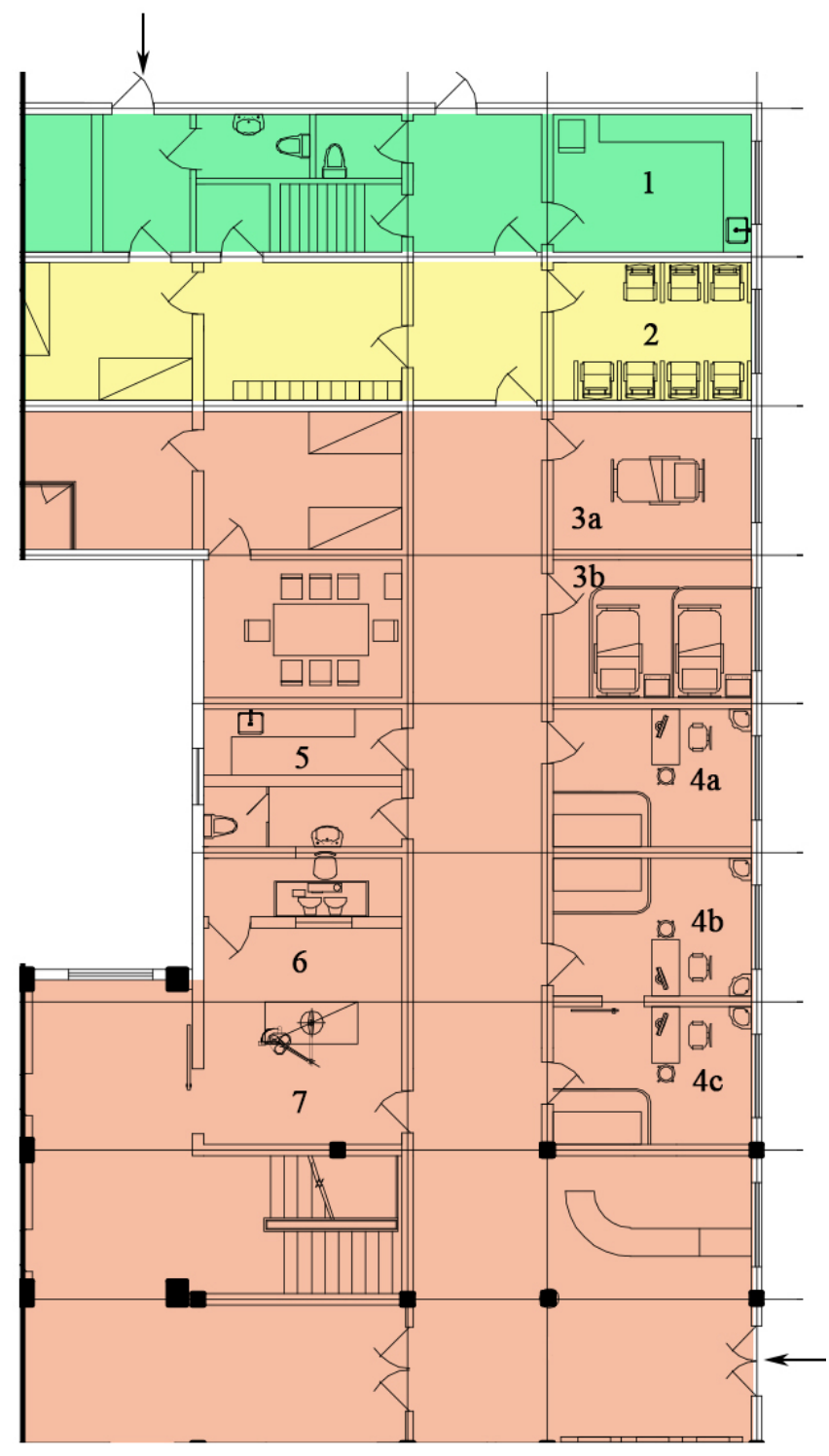

Figure 1 Layout of the old fever clinic in 2019. 12. [1] Cleaning area; [2] Half-contaminated area; [3a,3b] Observation rooms; [4a, 4b, 4c] Consultation rooms; [5] Laboratory room; [6] X-ray room; [7] Pharmacy.

the occurrence of the epidemic on the eve of Chinese Lunar New year, introduced major problems, as the construction personnel had left Shanghai on holiday, and the engineering materials market was mostly shut. Therefore, the hospital first secured the most basic settings and biosafety of the FUO emergency department.

Within 24 hours, the functional areas of the hospital had been divided according to the standard of "five insides", which means registration charge, inspection, examination, drug collection, and treatment could all be completed inside the FUO emergency department,. The area of 462 $\mathrm{m}^{2}$ was divided into three parts: receiving (contaminated area), dressing (semi-contaminated area); and living (clean area). Problems relating to registration were resolved using a system of self-service cost confirmation. Two consultation rooms, three independent observation rooms, and clinical laboratories to complete blood cell analysis, $\mathrm{C}$-reactive protein, influenza $\mathrm{A} / \mathrm{B}$ rapid screening were set up. The radiography room was adapted to accommodate digital radiology (DR) chest X-ray services. In addition, independent pharmacies were set up to provide symptomatic relief drugs (5).

More importantly, strict training and guidance, which anyone who enters the contaminated area has to take, was introduced by the Medical Affairs Department and the Nosocomial Infection Control and Management Office. Each medical worker must take three hours of independent training and pass an exam (6). Semi-contaminated areas and clean areas are equipped with full-time personnel, who ensure that protective equipment is worn and removed correctly, and two-way mirrors for self-inspection. Medical waste is promptly packaged and disposed of following the strictest double-layer isolation method (7).

\section{Vacating the ward and establishing observation areas}

According to the national standard of diagnosis and treatment, cases suspected by epidemiological history, clinical symptoms, and computed tomography (CT) images must be isolated and undergo observation while viral nucleic acid tests are conducted at the Centers for Disease Control and Prevention (CDC). Two nucleic acid tests with an interval of more than 24 hours are negative to rule out suspected cases (8). Therefore, each isolated case needs to stay in the hospital for a minimum of two days, and the observation room is finally disinfected after the patient leaves.

During the peak of the first outbreak in January, 2020, the three pre-existing observation rooms at Ruijin Hospital were soon unable to meet the retention needs. The hospital responded by turning an area of the vacated infection department into 12 independent wards for the treatment of suspected cases. The work was completed within 24 hours.

Infection physicians and physicians from other departments who are qualified to enter the isolation area are responsible for the admission of cases for observation. The transfer route from the FUO emergency department to the 
observation areas has been established, and independent passageways and elevators have been set aside.

\section{Expansion of the FUO emergency department and establishment of an adjacent observation area}

Temporarily, observation and treatment-related demands have been solved by vacating the ward of the infection department, although a number of problems still exist. First, the 600-meter moving path introduces risks during transportation, placing ordinary patients on other floors at risk of cross-infection. Second, in the absence of an independent central air conditioning system, the cold and humidity mean doctors and patients can easily catch colds.

Therefore, the expansion of the FUO emergency department by making use of the adjacent former outpatient finance office room became the primary task of the second stage. The work took only 11 days from decisionmaking to completion, 7 of which were Chinese New Year holidays. The hospital overcame the problems caused by material and personnel shortages, and within five days had demolished $206 \mathrm{~m}^{2}$ of space and installed 10 isolation wards, 1 nurse workstation, and 1 doctor workstation. The newly established areas were equipped with wireless network, electronic medical records (EMR) system, 12 rescue instruments, and fitted with the first remote ward-round robot used in an isolation ward in China. After the transformation, the total area of the FUO emergency department had been expanded to $668 \mathrm{~m}^{2}$.

\section{Setting up a fully equipped test and check-up patb and configuration scheme}

COVID-19 has atypical symptoms, long incubation period and rapid change of disease (9). When establishing a clinical pathway, using the limited examination methods efficiently and reducing the cross-infection risk to the minimum can present difficulty (10).

The expert group of Ruijin Hospital always has prioritized CT screening and detection of virus nucleic acid using reverse transcription-polymerase chain reaction (RTPCR) (11). When the original DR equipment could not meet the needs of diagnosis, the hospital, in cooperation with United Imaging, introduced a 16-slice CT for use specifically in the FUO emergency department. The hospital has achieved a balance between radiation dose and diagnostic efficiency by using the low-dose scanning protocol and $1.25 \mathrm{~mm}$ reconstruction parameter (12). The only problem that has arisen is the reconstruction of the scanner room.

Because of lead protection reinforcement, it usually takes four weeks to transform a DR scanner room into a CT scanner room. To complete this task in the given timeframe, the hospital's Radiology Department cooperated with the logistics department, using physical shielding and the existing lead protective door. When the CT is working, remote control allows the radiographers to operate the machine without exposure to radiation (13). Now, more than 100 chest CT scans can be performed every day.

In the middle of January 2020, Ruijin Hospital's virus laboratory participated in the development of the severe acute respiratory syndrome coronavirus 2 (SARS-CoV-2) nucleic acid detection kit with Bejing Genomics Institute (BGI). Nucleic acid detection was swiftly put into use and compared with the results of CDC. Considering the effectiveness of RT-PCR and the risk of virus particle concentration accumulation in the air of the RT-PCR amplification room, nucleic acid detection is not widely used for screening (14). By reforming the observation room and adding the biosafety cabinet, the rapid screening of virus latex agglutination can be carried out in the FUO emergency department, the detection time is shortened to 30 minutes, and the biosafety is effectively guaranteed (15).

At this point, the transformation of a totally enclosed FUO emergency department in Ruijin Hospital, a general hospital in China, has been completed. Four clinics and 12 observation rooms, with an independent pharmacy, CT scanner room, triage room and laboratory have been set up within $682 \mathrm{~m}^{2}$ (Figure 2). Three areas with strict isolation measures and paths can protect the safety of healthcare staff on duty to the maximum extent (16). Imaging and rapid virus screening can both be performed in the FUO emergency department.

\section{The innovative application of intelligent information technology}

The FUO emergency department is strictly isolated, but its operation requires many consultations and discussions. Streamlining the patient treatment process and improving the efficiency of diagnosis and treatment is dependent on a reliable information system (17). Before the reconstruction of Ruijin Hospital, $5^{\text {th }}$ generation $(5 \mathrm{G})$ network base stations were laid to support the use of intelligent information technologies. Those currently in use include:

(I) Welink Remote Video Consultation System: introduced on January 22 and used for the 


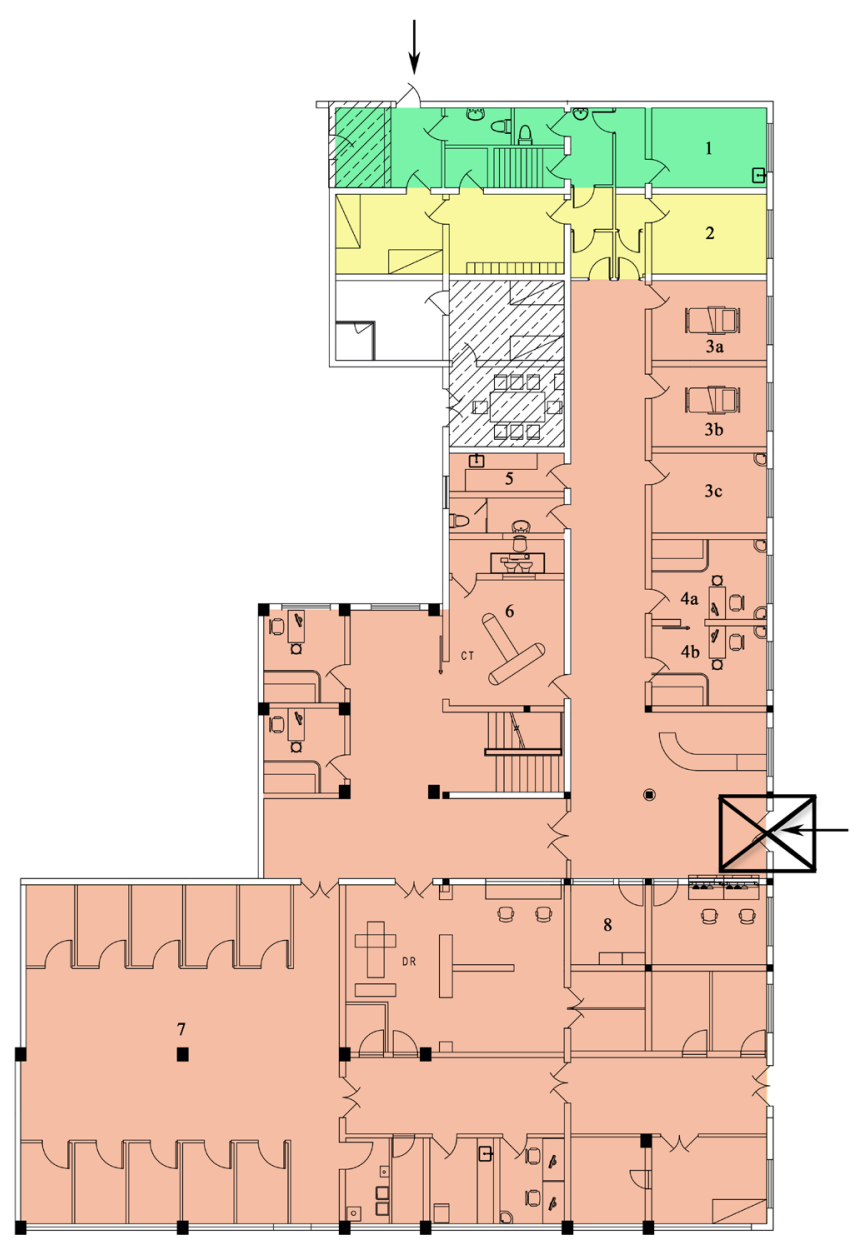

Figure 2 Layout of the rebuilt FUO emergency department. [1] Cleaning area; [2] Half-contaminated area; [3a, 3b, 3c] Observation rooms; [4a, 4b] Consultation rooms; [5] Laboratory room; [6] CT room; [7] Observation room.

consultation of the experts in the hospital and the attending physicians of the FUO emergency department;

(II) The mobile imaging module of the hospital's WeChat public account: permits online reading of CT images and laboratory reports on mobile phones;

(III) WeChat group: facilitates online discussion between all medical staff;

(IV) Head-mounted live broadcast system: facilitates remote ward rounds and communication with isolated observation cases;

(V) Noah logistics transportation robot: performs unmanned drug transportation;
(VI) Remote ward-round robot: allows for humanmachine dialogue with the isolated patients to help meet the observation requirements of physicians;

(VII) Artificial intelligence-aided voice follow-up system: a two-week voice follow-up is performed for the home observation cases, excluding those with COVID-19, and the voice is recorded and converted into text;

(VIII) Mobile infrared temperature measurement system: all healthcare staff entering the FUO emergency department undergo a real-time temperature measurement, and data is recorded automatically;

(IX) Fever-tracking system for healthcare workers: staff working in the FUO emergency department who have fever or a history of contact with epidemic areas must fill in and report online; they are tracked and followed up by the Medical Affairs Department, and electronic files are established.

These improvements and upgrades have created an intelligent closed-loop management system for pre-, midand post-diagnosis in the FUO emergency department. Under the epidemic situation of the novel coronavirus, an integrated and intelligent information system for the FUO emergency department has been built, forming a popularized brilliant information construction scheme for the emergency department.

\section{Effective allocation of human resources}

To accommodate the continuously changing guidelines of the Chinese government for disease prevention, control, diagnosis, and treatment, and based on empirical analysis by experts in 14 subjects, the supplies and manpower allocation of hospital have been dynamically adjusted to preserve the hospital's medical and human resources.

Doctors and nurses: The attending doctors of the infection and respiratory departments are attending physicians who are on duty every 24 hours. Two matrons in charge of coordinating the "three zones" are responsible for the polluted area and clean area, respectively, and ensure that medical staff in the polluted area work for no more than 8 hours a day, during which they can leave the polluted area once to rest and eat (18).

\section{Personal protective equipment}

On the premise of ensuring staff safety, the number of pieces of protective equipment is accurately calculated every 
day, the entry of additional personnel is prohibited, and measures to reduce the consumption of personal protective equipment are maximized.

\section{Technical team configuration and training in the FUO emergency department}

To better respond to the epidemic, improve work efficiency and diagnostic accuracy, save protective equipment, and preserve the standard of medical service, our hospital combined clinical expert remote video consultation with a mobile ward-round robot. This allows consultations and decision-making for "suspect" cases to be performed remotely and reduces the need for physicians to repeatedly enter the isolation area of the FUO emergency department, preserving their safety and reducing the consumption of unnecessary protective suits on the premise of ensuring the quality of consultations.

\section{Setting up the remote video system}

To improve the efficiency of consultation and to reduce the frequency of experts repeatedly entering the contaminated area, thus reducing the usage of protective suits, we installed a remote video system in a small conference room in the hospital. This facilitated direct communication with the doctor in the FUO emergency department via laptop and allowed electronic patient records to be transmitted through the hospital information system (HIS). Based on the imaging, test results, and contact history from the workstation, doctors decided whether to begin the screening procedure for novel coronavirus.

Establishing an expert group for the prevention and treatment of novel coronavirus pneumonia

An expert group was established based on the experience of Ruijin Hospital during the outbreaks of SARS, H7N9, and $\mathrm{H} 5 \mathrm{~N} 1$, including the associate chief physician and chief physician of the infectious diseases department, the respiratory and intensive care department, the hospital infection department, the intensive care department, and the emergency department. Radiologists of the same level performed imaging diagnosis (19). Physicians from two different departments served as in-hospital/district consultation experts. Experts undertook consultations of potential "suspect" cases raised by primary physicians and made an appropriate diagnosis. If opinions in diagnosis differed between the two experts, the case would be referred to the corresponding section director for consideration and diagnosis.

\section{Dynamic adjustment of the diagnosis and treatment scheme}

The hospital expert groups interpreted the "Diagnosis and Treatment Guidance of Novel Coronavirus Pneumonia" issued by the state and Shanghai City, and combined with the case review in the consultation, dynamically adjusted the "Diagnosis and Treatment Scheme of Ruijin Hospital", to create a suitable scheme for diagnosis, treatment, and prevention of COVID-19 in Shanghai (8).

\section{Training for all staff and strict prevention}

In consideration of the COVID-19 epidemic in January, 2020, the local government in Shanghai initiated its first-level response to public health events. Our hospital conducted online training for all staff regarding the scheme for diagnosis, treatment, and prevention created by the national and local governments and our hospital.

\section{Patient and public involvement}

This was a management improvement study and no patients were directly involved in the study design, in setting the research questions, or in the outcome measures. No patients were asked to advise on interpretation or the write up of results.

\section{Results}

After 14 days of reconstruction at Ruijin Hospital, the medical examinations and screening procedures relating to the COVID-19 outbreak could all be completed within the FUO emergency department. At present, the department is equipped with a dedicated 16-slice CT that can perform 150 cases of low-dose CT lung scanning per day. The laboratory is equipped with biosafety cabinets, which can carry out blood biochemical detection and latex agglutination tests for the SARS-CoV-2, and the results can be obtained within 40 minutes. There is also an independent pharmacy and treatment and waste disposal rooms. Medical staff are provided with living areas and necessary personal supplies for 24-hour duty. The whole febrile outpatient channel and path are entirely separate from the general ward and have an independent ventilation system.

Twelve isolated observation rooms have been fitted near the FUO emergency department, where 48-hour medial observation can be conducted. In addition, another isolation 
area (comprising 12 wards) in the Infectious Disease department has been prepared in case of emergency.

A rapid screening process for patients with fever was established, which mainly included epidemiological questionnaire, low-dose CT scan of lungs, blood biochemical examination [PCR, D-dimmer, complete blood count $(\mathrm{CBC})]$, and pharynx swab testing (influenza $\mathrm{A}$, influenza $\mathrm{B}$, and SARS-CoV-2 latex agglutination test) (Figure 3). The time between pre-checking a patient in the FUO emergency department and getting the result of initial virus screening has been reduced from 34 to 3 hours, the number of visiting steps has been decreased from 18 to 8 hours, and the time spent waiting for a consultation has been narrowed from 2 hours to 10 minutes.

The transfer path between the clinic, CT scanner room, and observation room has been shortened from 450 to $20 \mathrm{~m}$, and the observation time has been shortened from 72 to 26 hours. The median waiting time for image examination has been reduced from 40 to 3 minutes, and the moving distance has been shortened from 800 to $10 \mathrm{~m}$.

The diagnosis and treatment process covered by $5 \mathrm{G}$ network has been established, and there is zero contact between consultant physicians and patients, zero contact with logistics, and zero contact with surface disinfection of utensils.

From January 19, 2020 to 10 March 2020, the FUO emergency department received 2,863 cases, including 42 suspected cases and 8 confirmed cases of COVID-19 (Figure 4). Of the 8 confirmed cases, 2 cases were critical. All cases received timely and accurate screening and preliminary treatment, and 5 cases were discharged after transfer to a designated hospital for treatment.

At present, there are 46 doctors, 124 nurses, and 18 logistics staff working in the fever clinic, and 28 CDC sampling staff have so far come into contact with suspected and confirmed cases. Isolated observation and virologic examination has confirmed that no staff members have been infected.

\section{Discussion}

In the current COVID-19 epidemic, as with the SARS outbreak of 2003, nosocomial transmission has proved to be a key way in which disease is transmitted (20). Close contact with virus carriers with atypical symptoms, nursing of confirmed cases, and timely intubation, respiratory support, and rescue treatment for critically ill patients are all necessary tasks for medical staff. However, these duties also present the risk of exposure to infection (21). Even more troublesome is cross-infection between teams of medical staff and the wider spread from inside to outside the hospital. Treatment teams must conduct many internal discussions, and severe cases require the joint decisionmaking and treatment planning of the multidisciplinary diagnosis and treatment team (MDT) (22). Members of the medical team who come into contact with suspected or confirmed cases are, therefore, an important potential source of infection (23). Moreover, patients with pneumonia must undergo imaging, laboratory tests, diagnostic procedures, and even pathological sampling and other necessary traumatic treatments (24). Therefore, as part of the regular diagnosis and treatment process, there is a flow of doctors and patients between different departments and different buildings of the hospital. The early cases of nosocomial infections seen in Wuhan were typical (25). In order to minimize the probability of nosocomial infection and to provide our patients with timely diagnosis and treatment, it is very important to use the lean management model to deploy manpower and material resources and to make dynamic adjustments when necessary. Herein, we formulate a realistic process, in which we reconstructed the FUO with 40-years' history into a standardized FUO emergency department in a short period of time under the lean management. We were able to summarize our implementation into three main steps, and share detailed measures and design management all the way from facilities to personnel. With lean management, the quick reaction, comprehensive cross-department cooperation, as well as the dynamic adjustment can be ensured against COVID-19 outbreak. Of note, we made innovative application of intelligent information technology based on $5 \mathrm{G}$ networks during the epidemic, with the help of which, doctors and patients with highly contagious status such as COVID-19 infection can achieve zero contact, thus reducing chances of cross-infection.

This experience of employing the lean management model could also be carried out in secondary hospitals. The main factor for success of this model lies in adoption of the lean management and cooperation in and across departments. Secondary hospitals are usually the first stop for inspection of suspected COVID-19 cases. In addition, our experiences in reconstructing and standardizing a FUO followed the basic rules and recommendations from the CDC. It could function like a modeled scenario. And some of the information technology, such as WeChat and remote video consultation were easy to access. Therefore, although 


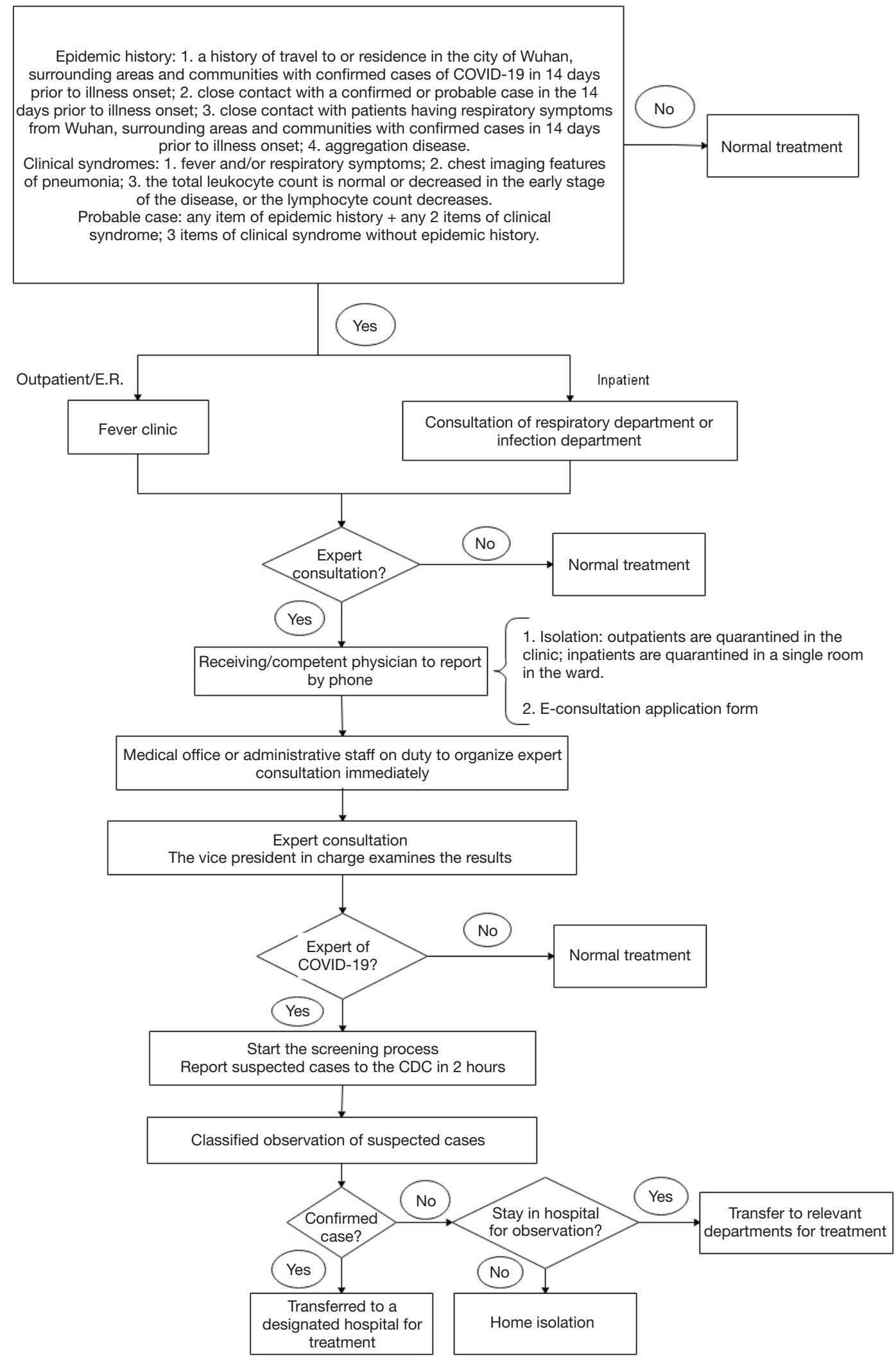

Figure 3 Workflow of diagnosis and consultation of febrile patients. 


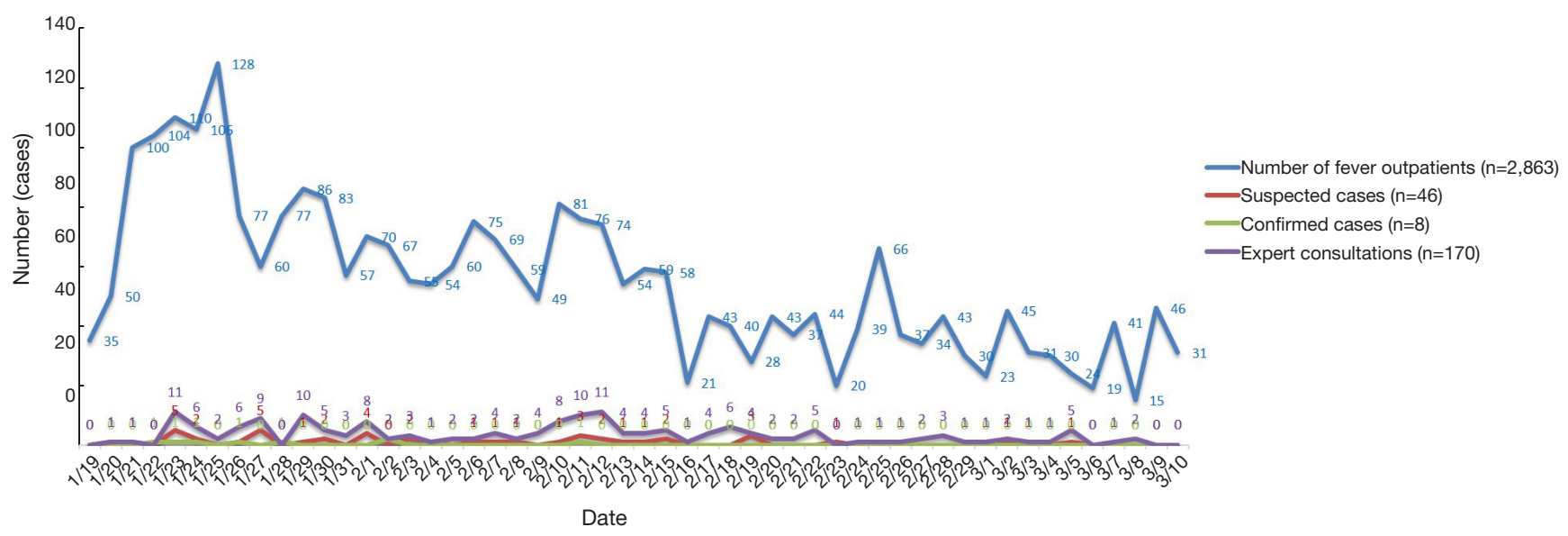

Figure 4 Workload of the FUO emergency department in Ruijin Hospital from 00:00 19 January 2020 to 24:00 10 March 2020.

materials might be relatively limited, the implementation of this lean management model is also thought suitable for secondary hospitals.

With its large scale and high population density, general hospitals in China are the busiest medical institutions in the world. The number of outpatients in public hospitals in large Chinese cities is 18 times that of American hospitals with the same bed capacity, while the numbers of emergency and surgery patients, respectively, are about three and four times those in equivalent American institutions. In China, because of the population density, the establishment of "safe zones" in hospitals has become the key to controlling the spread of infectious diseases.

\section{Strengths and weaknesses of this study}

Since the outbreak of COVID-19, Ruijin Hospital Affiliated to the School of Medicine of Shanghai Jiaotong University has established a standardized FUO emergency department in a short period of time (26). Through the rapid establishment of isolation zones, exceptional duties and protective preparation, and an Internet and technologybased follow-up consultation system, a front-line station to receive and identify suspected cases of COVID-19 has been set up.

On-the-spot transformation is the basic principle for improving the capability of a hospital to receive patients. It can open up the existing emergency or relatively independent outpatient areas, transforming them into a separate area for receiving and observing patients. The standard of "five insides" is the key to a temporary FUO emergency department. "Five insides" refers to the re-division of the functional area so that registration, inspection, CT examination, pharmacy, and treatment are dealt with in the isolated area." According to the biosafety requirements, the entire area is divided into three parts: the pollution zone, the buffer zone, and the clean zone. Unnecessary entrances, exits, and interregional channels are padlocked. It is suggested that all three districts should be guarded by personnel at all times to avoid accidental entry and to ensure protective clothing is worn correctly $(27,28)$.

The number of isolated observation rooms can be determined according to the time of indwelling observation. In addition to essential ward equipment, necessary first aid equipment and medicine should also be provided. Since January 19, 2020, the FUO emergency department of Ruijin Hospital has received more than 2,600 cases, including 38 suspected cases, which have been ruled out. Another 8 cases were confirmed, including two critical cases, and no medical staff has been infected. However, it may be related to the small number of patients who were actually infected. But effective isolation and observation and strict personal protection play a more important role in defending medical staff from infection. The number of our staff members has been correspondent to the average in-patient number. And the supplies and manpower allocation of the hospital have been dynamically adjusted accordingly. It should be noted that the principle of retention observation is that confirmed cases can be quickly transferred to designated hospitals after rapid screening, and fever cases with COVID-19 can be admitted or returned home for observation in time. Among the emergency cases mentioned above, we also found a variety of cases of influenza, encephalitis, peritonitis, and acute myocardial infarction. 
The necessary tests for febrile cases include epidemiological investigation, low-dose CT lung scanning, biochemical blood tests, and rapid screening of viral throat swabs (29). The decrease in the number of visits shortened waiting time drastically, which is also the key in determining the indwelling observation time. When cases are very concentrated, clinically confirmed cases based on symptoms and CT manifestations are also useful. However, this method is limited to areas where the spread is rapid (30). Unless direct contact with the patient is necessary, access to the FUO emergency department must be kept to a minimum. Internet and technology can also support this, by facilitating remote multidisciplinary consultation, drug logistics robot, and artificial intelligence follow-up (31).

\section{Conclusions}

When the COVID-19 outbreak first occurred, Chinese hospitals faced many challenges. Our experience of employing the lean management model could quickly implement effective outpatient prevention and control, and is worthy of reference for countries around the world in dealing with COVID-19 and other fulminant respiratory infectious diseases.

\section{Acknowledgments}

Funding: The study was supported by The Special subject of novel coronavirus's Prevention and Control, Shanghai Jiaotong University (2020RK66); Correlation Between MRI characteristics of articular cartilage under different exercise loads and differentiation of chondrocytes into HTCs-B (HKM201901); Special subject of Integrated traditional Chinese and Western Medicine in Shanghai General Hospital (ZHYY-ZXYJHZX-201901).

\section{Footnote}

Conflicts of Interest: All authors have completed the ICMJE uniform disclosure form (available at http://dx.doi. org/10.21037/atm-20-4329). The authors have no conflicts of interest to declare.

Ethical Statement: The authors are accountable for all aspects of the work in ensuring that questions related to the accuracy or integrity of any part of the work are appropriately investigated and resolved. The study was approved by Ruijin Hospital affiliated to School of
Medicine, Shanghai Jiaotong University.

Open Access Statement: This is an Open Access article distributed in accordance with the Creative Commons Attribution-NonCommercial-NoDerivs 4.0 International License (CC BY-NC-ND 4.0), which permits the noncommercial replication and distribution of the article with the strict proviso that no changes or edits are made and the original work is properly cited (including links to both the formal publication through the relevant DOI and the license). See: https://creativecommons.org/licenses/by-nc-nd/4.0/.

\section{References}

1. National Health Commission of the People's Republic of China. Guidelines for setting up fever (emergency) clinics in medical institutions (try-out). 2003

2. Li Q, Guan X, Wu P, et al. Early Transmission Dynamics in Wuhan, China, of Novel Coronavirus-Infected Pneumonia. N Engl J Med 2020;382:1199-207.

3. She J, Jiang J, Ye L, et al. 2019 novel coronavirus of pneumonia in Wuhan, China: emerging attack and management strategies. Clin Transl Med 2020;9:19.

4. Wang J, Zhou M, Liu F. Exploring the reasons for healthcare workers infected with novel coronavirus disease 2019 (COVID-19) in China. J Hosp Infect 2020. DOI: https://doi.org/10.1016/j.jhin.2020.03.002.

5. Huang C, Wang Y, Li X, et al. Clinical features of patients infected with 2019 novel coronavirus in Wuhan, China. Lancet 2020;395:497-506.

6. Huh S. How to train health personnel to protect themselves from SARS-CoV-2 (novel coronavirus) infection when caring for a patient or suspected case. J Educ Eval Health Prof 2020;17:10.

7. WHO. Water, sanitation, hygiene and waste management for COVID-19: technical brief, 03 March 2020.

Available online: https://apps.who.int/iris/bitstream/ handle/10665/331305/WHO-2019-NcOV-IPC_WASH2020.1-eng.pdf

8. National Health Commission of the People's Republic of China. Prevention and Control Plan of coronavirus disease 2019 (COVID-19) (Sixth Edition) (Internet). 2020. Available online: http://www.nhc.gov.cn/xcs/zhengcwj/202 002/8334a8326dd94d329df351d7da8aefc2/files/b218cfeb1 bc54639af227f922bf6b817.pdf

9. Sun P, Lu X, Xu C, et al. Understanding of COVID-19 based on current evidence. J Med Virol 2020. [Epub ahead of print]. 
10. Chen S, Yang J, Yang W, et al. COVID-19 control in China during mass population movements at New Year. Lancet 2020;395:764-6.

11. Ai T, Yang Z, Hou H, et al. Correlation of chest CT and RT-PCR testing in coronavirus disease 2019 (COVID-19) in China: A report of 1014 cases. Radiology 2020:200642. [Epub ahead of print].

12. Li Y, Xia L. Coronavirus Disease 2019 (COVID-19): Role of Chest CT in Diagnosis and Management. AJR Am J Roentgenol 2020:1-7. [Epub ahead of print].

13. Kanne JP, Little BP, Chung JH, et al. Essentials for Radiologists on COVID-19: An Update-Radiology Scientific Expert Panel. Radiology 2020:200527. [Epub ahead of print].

14. WHO. Laboratory testing for coronavirus disease 2019 (COVID-19) in suspected human cases. 2020 March. Available online: https://apps.who.int/iris/bitstream/ handle/10665/331329/WHO-COVID-19-laboratory2020.4-eng.pdf

15. WHO. Laboratory biosafety guidance related to coronavirus disease 2019 (COVID-19): interim guidance, 12 February 2020. Available online: https://apps.who.int/ iris/bitstream/handle/10665/331138/WHO-WPE-GIH2020.1-eng.pdf

16. Wu Z, McGoogan JM. Characteristics of and Important Lessons From the Coronavirus Disease 2019 (COVID-19) Outbreak in China: Summary of a Report of 72314 Cases From the Chinese Center for Disease Control and Prevention. JAMA 2020. [Epub ahead of print].

17. Zhai Y, Wang Y, Zhang M, et al. From Isolation to Coordination: How Can Telemedicine Help Combat the COVID-19 Outbreak? medRxiv 2020. doi: https://doi.org /10.1101/2020.02.20.20025957.

18. Xie J, Tong Z, Guan X, et al. Critical care crisis and some recommendations during the COVID-19 epidemic in China. Intensive Care Med 2020. [Epub ahead of print].

19. Zhang S, Diao MY, Duan L, et al. The novel coronavirus (SARS-CoV-2) infections in China : prevention, control and challenges. Intensive Care Med 2020;46:591-3.

20. Guarner J. Three Emerging Coronaviruses in Two Decades: The Story of SARS, MERS, and Now COVID-19. Am J Clin Pathol 2020;153:420-1.

21. Bai Y, Yao L, Wei T, et al. Presumed Asymptomatic Carrier Transmission of COVID-19. JAMA 2020;323:1406-7.
22. Calisher C, Carroll D, Colwell R, et al. Correspondence: Statement in support of the scientists, public and medical professionals of China. Lancet 2020;395:e42-3.

23. Hellewell J, Abbott S, Gimma A, et al. Feasibility of controlling COVID-19 outbreaks by isolation of cases and contacts. Lancet Glob Health 2020;8:e488-96.

24. Chen N, Zhou M, Dong X, et al. Epidemiological and clinical characteristics of 99 cases of 2019 novel coronavirus pneumonia in Wuhan, China: a descriptive study. Lancet 2020;395:507-13.

25. Chen X, Tian J, Li G, et al. Initiation of a new infection control system for the COVID-19 outbreak. Lancet Infect Dis 2020;20:397-8.

26. Peeri NC, Shrestha N, Rahman MS, et al. The SARS, MERS and novel coronavirus (COVID-19) epidemics, the newest and biggest global health threats: what lessons have we learned? Int J Epidemiol 2020. [Epub ahead of print].

27. WHO. Key planning recommendations for mass gatherings in the context of the current COVID-19 outbreak: interim guidance, 14 February 2020. Available online: https://apps.who.int/iris/bitstream/ handle/10665/331502/WHO-2019-nCoVPOEmassgathering-2020.2-eng.pdf

28. Peng J, Ren N, Wang M, et al. Practical experiences and suggestions on the eagle-eyed observer, a novel promising role for controlling nosocomial infection of the COVID-19 outbreak. J Hosp Infect 2020;105:106-7.

29. Sohrabi C, Alsafi Z, O’Neill N, et al. World Health Organization declares global emergency: A review of the 2019 novel coronavirus (COVID-19). Int J Surg 2020;76:71-6.

30. Fang Y, Zhang H, Xie J, et al. Sensitivity of Chest CT for COVID-19: Comparison to RT-PCR. Radiology 2020. [Epub ahead of print].

31. Dong E, Du H, Gardner L. An interactive web-based dashboard to track COVID-19 in real time. Lancet Infect Dis 2020;20:533-4.

Cite this article as: Chen T, Ma X, Zhou S, Wang H, Pan Y, Chen L, Lv H, Lu Y. Establishing a standardized FUO emergency department: design and practice in dealing with COVID-19. Ann Transl Med 2020;8(12):749. doi: 10.21037/ atm-20-4329 